\title{
Tonda, Joseph. - La guérison divine en Afrique centrale (Congo, Gabon)
}

Paris, Karthala (« Hommes et sociétés »), 2002, 243 p.

\section{Emmanuelle Kadya Tall}

\section{(2) OpenEdition}

\section{Journals}

Édition électronique

URL : http://journals.openedition.org/etudesafricaines/4886

DOI : 10.4000/etudesafricaines.4886

ISSN : 1777-5353

\section{Éditeur}

Éditions de l'EHESS

Édition imprimée

Date de publication : 20 décembre 2004

Pagination : 982-985

ISBN : 978-2-7132-2005-0

ISSN : 0008-0055

\section{Référence électronique}

Emmanuelle Kadya Tall, «Tonda, Joseph. - La guérison divine en Afrique centrale (Congo, Gabon)»,

Cahiers d'études africaines [En ligne], 176 | 2004, mis en ligne le 17 avril 2008, consulté le 22 septembre 2020. URL : http://journals.openedition.org/etudesafricaines/4886 ; DOI : https://doi.org/10.4000/ etudesafricaines. 4886

Ce document a été généré automatiquement le 22 septembre 2020

(c) Cahiers d'Études africaines 


\title{
Tonda, Joseph. - La guérison divine en Afrique centrale (Congo, Gabon)
}

Paris, Karthala (« Hommes et sociétés »), 2002, 243 p.

\author{
Emmanuelle Kadya Tall
}

1 L'ouvrage de Joseph Tonda est rafraîchissant à plus d'un égard. Livrant une véritable croisade contre les ethnocentrismes et culturalismes en tout genre, il souhaite en finir avec les oppositions binaires (tradition/modernité, paganisme/christianisme) et propose un concept qui va lui permettre d'analyser ensemble des phénomènes d'ordre religieux, thérapeutique et politique au Congo et au Gabon : le Souverain moderne. Par ces termes l'auteur veut "désigner la puissance hégémonique de la mission civilisatrice, au nom de laquelle s'est légitimée de façon ultime la "modernisation" en Afrique » (p. 22).

2 Critiquant les analyses ethnocentriques qui cherchent à « sortir le Dieu chrétien ou le Souverain moderne des structures de causalité du malheur en Afrique» (p. 39), Joseph Tonda émet la thèse selon laquelle « le Dieu civilisateur et le Génie sorcier, en tant que significations imaginaires sociales, et donc marquées par l'ambivalence, ne se définissent pas dans un rapport d'extériorité irréductible en Afrique... C'est une même puissance que symbolisent Dieu, le Génie sorcier, et la coalition des forces qui le soutiennent dans la "modernité africaine", c'est-à-dire le système capitaliste et chrétien» (p. 39). Replaçant ainsi la logique sorcellaire dans un rapport dialectique avec les logiques chrétienne et capitaliste, l'auteur rend caduques toutes les analyses sociologiques de la sorcellerie en termes de paganisme ou de re-traditionnalisation.

3 Après une introduction fort brillante, où sont posées d'emblée ses hypothèses et les auteurs sur lesquels il s'appuie (principalement Marx, Weber et Bourdieu), Joseph Tonda met à l'épreuve, en sept chapitres et angles d'approche différents, son concept de Souverain moderne. Dès le premier chapitre, nous comprenons que les « affaires du corps ", c'est-à-dire la maladie et l'infortune ont partie liée avec le capital de Dieu et qu'il est vain de penser la sorcellerie comme étant extérieure au travail de Dieu. Le nœud gordien de sa démonstration nous semble nicher dans le chapitre 2 qui traite de " Mademoiselle ${ }^{1}$ et Mami Wata » : le chapitre précédent comme les suivants renvoient 
constamment à ces deux figures symboliques. Mademoiselle comme avatar de la Vierge Marie et Mami Wata, figurant en quelque sorte son double diabolique, sont en relation spéculaire et sont complémentaires l'une de l'autre, réunissant ensemble ce qui définit le Souverain moderne, à savoir, la figure du Christ, la Bible en tant qu'écriture, l'État colonial et postcolonial, l'argent et les marchandises. Dans le chapitre 3, l'auteur nous explique que «le syndrome du prophète-guérisseur » n'est pas l'apanage de ceux que l'on dénomme tradipraticiens selon la terminologie de l'oms. Tous ceux qui ont partie liée au pouvoir individualisant de l'écriture y sont confrontés. «La connaissance fondée sur l'écriture apparaît ici comme la "force productive" la plus importante des identités modernes. C'est la raison pour laquelle elle donne lieu à des processus d'appropriation symbolique par la médiation desquels des agents sociaux travaillent à affirmer, par l'imaginaire, leurs identités. [...] Il est donc difficile de considérer comme fortuit le fait que ces processus soient liés à des prophétismes thérapeutiques et antisorciers. Produit par excellence des logiques individualistes propres à la situation de crise d'identité à laquelle il prétend mettre fin, le prophète tente de reconstituer la fonction totalisante $\mathrm{du}$ champ thérapeutique et religieux indigène [...] en recomposant les fonctions $d u$ prêtre et du médecin séparées par la biomédecine et l'Église missionnaire tout en inscrivant son action dans une perspective individualisante » (pp. 106-107).

Dans le chapitre 4, "Guerre des esprits, des sexes et des classes d'âge autour de la guérison divine ", Joseph Tonda décrit à partir d'exemples concrets (Mademoiselle et le Ngol) comment le Souverain moderne a déstructuré les rapports sociaux de sexe et de classe d'âge au Congo et au Gabon. On peut regretter que l'auteur n'ait pas mis en parallèle ces mouvements centre-africains avec des mouvements et cultes similaires (nous pensons notamment au culte Deima en Côte-d'Ivoire analysé par Denise Paulme ${ }^{2}$, ou encore au culte Atinga au Nigeria analysé par P. Morton-Williams ${ }^{3}$ et revisité par A. Apter ${ }^{4}$ ) qui sont eux aussi des créations répondant à l'hégémonie du Souverain moderne. Dans le chapitre suivant sur « l'écriture et la guérison divine du sida ", Joseph Tonda insiste sur l'équation écriture, puissance hégémonique des Blancs et modernité. Ainsi les médecins non biomédicaux qui s'approprient la connaissance du sida ne sont pas de vieux tradipraticiens illettrés mais bien au contraire de jeunes lettrés urbanisés. Dans le chapitre 6 sur les "parcours de guérison et de conversion», Joseph Tonda reconnaît l'existence d'un véritable travail syncrétique (cf. J.-P. Dozon et A. Mary) opérant entre des schèmes de pensée produits par une socialisation traditionnelle entrant en affinité symbolique avec les schémas explicites du travail de Dieu sur le Mal, mais ce travail syncrétique "qu'ils [les travailleurs de Dieu] réalisent et dont la médiation fondamentale est constituée des affinités symboliques entre logiques païennes et logiques chrétiennes ne saurait invalider leur qualité de chrétiens, puisqu'il le commande » (p. 179). Enfin, dans un dernier chapitre sur «la violence politique du Souverain moderne au Congo ", l'auteur tente de rapprocher l'essor des mouvements de guérison divine depuis les années 1980 et, le processus de décomposition de l'ordre politique, économique, social et culturel dont témoigne la chasse aux sorciers, généralisée à la fin du régime du Parti unique. Les affaires du corps et les affaires politiques se trouvent en situation de fusion-superposition, «les uns et les autres agissant à la fois selon un "esprit" bureaucratique et surtout un ethos de la persécution » (p. 199).

5 La grande force de l'ouvrage de Joseph Tonda tient à cette idée de rupture ${ }^{5}$ dans les schèmes symboliques de pensée à partir de l'irruption du Souverain moderne et de son intégration dans les structures de causalité du malheur en Afrique. Ainsi prophètes- 
guérisseurs, pasteurs des Églises nouvelles, chefs de culte " néotraditionnels » sont tous travaillés par Dieu et la mission civilisatrice dont il est en quelque sorte le grand commandeur. Le titre original de l'ouvrage le travail de Dieu, refusé par l'éditeur sous un prétexte commercial puéril, exprime bien cette idée de la puissance du Dieu monothéiste. Mais un autre Dieu monothéiste est singulièrement absent dans l'ouvrage de Joseph Tonda. Le Dieu des musulmans a lui aussi travaillé les schèmes symboliques de pensée autochtones dans les régions d'Afrique où il s'est diffusé, et ce bien avant la mission civilisatrice chrétienne : en effet, la diabolisation du sorcier se retrouve bien présente dans les sociétés islamisées et, la "magification » de l'écriture y est poussée à son comble puisque l'on y boit les versets coraniques.

Le reproche dont Joseph Tonda pourrait faire l'objet est celui d'avoir circonscrit un projet théorique somme toute assez ambitieux, aux seuls exemples congolais et gabonais. Cette modestie de l'auteur participe d'une rigueur et honnêteté intellectuelle certaine, toutefois, on pourrait aussi y voir un certain penchant «afrocentralisé ». En effet, Mami Wata et le culte de Mademoiselle rappellent d'autres cultes, d'autres récits mythiques ailleurs en Afrique et nous irons jusqu'à dire, dans le vaste monde. Des personnages mythiques ou légendaires y ont vu le jour dans les mêmes conditions socio-historiques de colonisation des corps et des esprits. Ainsi, Mami Wata est largement diffusée dans toute l'Afrique occidentale et elle fait même l'objet d'un culte sur l'ancienne Côte des Esclaves. Des comparaisons plus larges auraient sans nul doute donné plus de poids à la portée universelle que le concept de Souverain moderne ne manque pas d'avoir. Une fois encore, on ne peut s'empêcher de penser que Joseph Tonda est lui aussi victime d'un certain travers culturaliste, en s'empêchant de rebondir sur des exemples qui ne font pas partie de l'aire culturelle bantoue. Dans le même ordre d'idée, il aurait été intéressant de voir se confronter l'islam et le christianisme, en retenant que la puissance du christianisme avait été renforcée par la nature hégémonique du capitalisme, modèle socio-économique dont il serait issu.

\section{NOTES}

1. Culte antisorcellerie de forte inspiration chrétienne.

2. D. PAulme, «Une religion syncrétique en Côte-d'Ivoire. Le culte Deima », Cahiers d'études africaines, $\mathrm{n}^{\circ}$ 9, 1962, pp. 5-90.

3. P. MoRTON-Williams, « The Atinga Cult among the South-Western Yoruba: A Sociological Analysis of a Witch-Finding Movement », Bulletin de l'IFAN, 18, $\mathrm{n}^{\circ}$ 3-4, 1956, pp. 315-334.

4. A. APTER, « Atinga Revisited : Yoruba Witchcraft and the Cocoa Economy, 1950-1951 », in Jean and John ComARofF (dir.), Modernity and its Malcontents. Ritual and Power in Postcolonial Africa, Chicago, University of Chicago Press, 1993, pp. 111-128.

5. Référence à Yves BAREL, Le paradoxe et le système, Grenoble, PUG, 1979. La rupture avec le système étant un lien, qui est notamment un lien d'adhésion, dont les Églises pentecôtistes constituent un vecteur particulièrement performant (Tonda, p. 92). 